\title{
DEVELOPMENT OF ALOGORITHM TO RECOGNIZE THROUGH SLOT FEATURE FROM STEP AP224 NEUTRAL FILE
}

\author{
Ashok G' ${ }^{1}$, S S Hebbal ${ }^{2}$, Sachhidanand Reur ${ }^{3}$ \\ ${ }^{1}$ Associate Professor, Mechanical Engineering, BKIT, Bhalki \\ ${ }^{2}$ Professor, Industrial \& Production Engineering, P D A College of Engineering, Kalburgi \\ ${ }^{3}$ Professor, Industrial \& Production Engineering, P D A College of Engineering, Kalburgi
}

\begin{abstract}
This research work aims at the recognition of through slot feature from a CAD part model using its data exchange file STEP AP224 of ISO 10303 standards. This is a part of ongoing research work for developing a program for Computer Aided process planning to link CAD and CAM. The feature is identified by developing algorithm or procedure, which can be implemented by a computer program.
\end{abstract}

Keywords: Feature, STEP, CAD, CAM, CAPP, Data exchange.

\section{INTRODUCTION}

The manufacturing industries are facing stiff competition because of globalization and to deliver high quality products at reasonable cost. The manufactures are forced to go for automation of their manufacturing facilities. The automation helps the industries to manufacture the products at lower cost, reduced manufacturing lead time, reduced material wastage, reduced time to delivery products, increase product quality, improved productivity, less time in administrative activity and reduced errors, etc. The manufacturing facilities are automated by using computer hardware and software's. The automation mainly involves, integration of design and manufacturing process through process planning using computers, I,e transfer of product design data from CAD to CAPP system and from CAPP to CAM system seamlessly. This direct transmission of data between design, process plan and manufacturing is not possible, because the data in CAD system is in proprietary format and which cannot be directly transmitted to the CAPP system for the reason said above.

Every CAD system stores product design data in its database, in the proprietary format; this creates a data exchange problem between different computer aided systems for exchange of data between different computer aided systems. To overcome this problem, there should be one file format which is common to all CAD systems for data exchange between different computer aided systems, so this common file format is called neutral file. A neutral file is one which is independent of any computer aided system. There are number of neutral file already exists, like DXF, IGES, etc. Most of these are very descriptive, lengthy, lack performance and consistency and they are not used much at present. These files are not common to all CAD software's available in world, some are supporting and some may not. We need a neutral file which is common to almost all CAD software's and provide much details about the product keeping in view the product life cycle and help in data exchange. Looking into the problems arise because of proprietary formats, the International Organization for Standardization (ISO) has developed a neutral file called STEP (Standard for Exchange of Product model data), which is adopted by all most all CAD systems in the world today and is also accepted by world manufacturing community.

This neutral file is best suited for data exchange and for integration of CAD and CAM through CAPP, because it is independent of any CAD system available in the world. The STEP is published in number of series, for each application, one series is applicable and these are called application protocols. The Application protocols are, 203,214, etc, for our research work the application protocol AP224 is used, which is exclusively designed for computer aided process planning (CAPP).

The present research work involves recognition of features from the neutral file STEP AP 224 for the development of CAPP system to link CAD and CAM. In this research paper, feature through slot is recognized from STEP AP224 neutral file by developing procedures. The details are presented in following section.

\section{LITERATURE SURVEY}

The design specifications of a product are converted into manufacturing instruction through process plan. Using process plan the product is manufactured accordingly. Process plan is an important activity; this whole activity of process plan has to be automated, to automate manufacturing facilities. The process plan activity can be automated by developing a computer program to read the description of product in the neutral file and analyze the neutral file for the profile of the product by recognizing its features. Feature recognition is one of the important tasks in the process planning which makes the program to understand the profile or configuration of the product. There 
are number of researcher who have reported their work in feature recognition, automated process planning development, integration of CAD and CAM through CAPP, etc. all these works focus on recognition of feature by developing various algorithms or procedures which are implemented through computer programs. S. Nagarajan et.al developed a system which is independent of hardware and software of the system and identifies design, manufacturing and interacting features along with access direction and volume to be removed from the feature, using lower level I,e B-Rep geometry of the neutral file STEP AP 203. The developed system is limited to only prismatic parts. Patrick Martin et.al, have recognized the slot feature from STEP neutral file and made an evaluation of slot feature for manufacturability by using different machining processes. Jung Hyun Han et.al, have developed a system which identifies only manufacturable features by using cutting tool database and formed an feature dependency structure. They have applied an algorithm on dependency structure to identify optimal machining sequence. Jaider Oussama, et.al, have developed a system which identifies isolated and interactive features of rotational parts using ISO standard STEP Application Protocol 203 Ed2 neutral file. From the combination of identified isolated features they have generated interacting features. Hyun Chan Lee, et.al, have identified interactive features directly from CAD data by an projective feature recognition algorithm proposed by the authors. These identified features they have used in process planning to generate process sequence and to minimize number of setup orientations. C. F. Tan, has developed a Hole Recognition System for CAPP using a neutral data format produced by CAD system. The geometrical data of holes is retrieved from STEP. Rulebased algorithm is used during recognising process. Chen Wong Keong, et.al, have designed a system which takes the neutral file ISO $10303-203$ as input, which recognizes features and extracts information related to features through database designed. The extracted information is used for manufacturing purpose. Sreenivasulu Reddy, et.al, has developed a JAVA program for feature identification. The input data for the program is in STEP neutral file. The program has been developed for extracting geometric data from the neutral file. It recognizes the features of a part based on geometric reasoning approach by creating link with B-rep database. P. Arunkumar, et.al, have developed a program to extract the features of a product from CAD model using database and modelling tools from STEP AP203. Most effort had been put to extract the product and for determining the sequence of operations to be performed. Jami J. Shah, et.al, have discussed the past 25 years of research in feature recognition, the discussion has focused on more successful ones. These include graph based, hint based, convex hull decomposition and volume decomposition - recomposition techniques. Recent advances in recognizing features with free form features are also presented. In order to benchmark these methods, a frame of reference is created based on topological generality, feature interactions handled, surface geometry supported, pattern matching criteria used, and computational complexity. V. N. Malleswari, et.al, have developed a program in JAVA, to identify cylinder and cross hole feature, using rule based method for a rotational part from STEP AP 203 neutral file. D. Sreeramulu, et.al, have written an computer program in JAVA language to identify features of rotational parts from neutral file. They have also proposed to send this extracted data to STEP-NC for seamless integration of CAD,CAPP and CAM. Yingguang Li, et.al, have defined and implemented a new type of feature called 'rib' which is commonly used aircraft structural parts. Grzegorz Nikiel, have introduced, an automatic procedure for conversion of the rotational parts $3 \mathrm{D}$ model to the $2 \mathrm{D}$ model and the $\mathrm{B}$ Rep model representation method is applied and a STEP file (ISO 10303:203) as the data carrier is used.

The above literature survey shows most of the researchers have reported their research work done in the domain of feature recognition using STEP neutral file. Majority of the work are carried, using the STEP application protocol AP203, as it is based on B-Rep and needs very complex algorithms to recognize the features. On the other hand the application protocol AP-224, provides significant information about the features, part and administrative activities, for the current research work this application protocol has been used for recognition of through slot feature.

\section{STEP DATA EXCHANGE FORMAT WITH}

\section{AP224}

STEP provides a standardized method to define all data which are necessary for the description of the CAD model throughout its life cycle. STEP is an ASCII file and provides product information along with mechanism and definition for product data exchange and sharing among dissimilar Computer Aided (CAx) platforms. STEP standard categorizes CAD data based on Application Protocols (AP). APs are used to define the collection of information required for the base exchange unit. Each AP is known as a formal document that describes the activities of the lifecycle of a CAD model. Currently, there are a few APs that are actively used in research and industries. For examples, AP 201 is explicit drafting, AP 203 for 3D design and the latest one, AP 238 is for STEP-NC manufacturing. This work is a part of an ongoing research work on development of software for process planning and to integrate the CAD and CAM through this developed CAPP software. The application protocol used for the development of CAPP is AP 224, because it provides complete manufacturing information apart from geometrical and topological information to machine single piece mechanical parts and it is exclusively designed for CAPP. STEP AP224 defines data models which are ranging from low level geometrical definition to high level product definition. The topology and geometrical information of B-Rep model relates to low-level definition and data related to manufacturing and machining feature are related to high level product definition.

\section{SYSTEM ARCHITECTURE}

The figure below shows the architecture of the system, which is self explanatory and shows how data flows seamlessly from CAD to CAM through CAPP. 


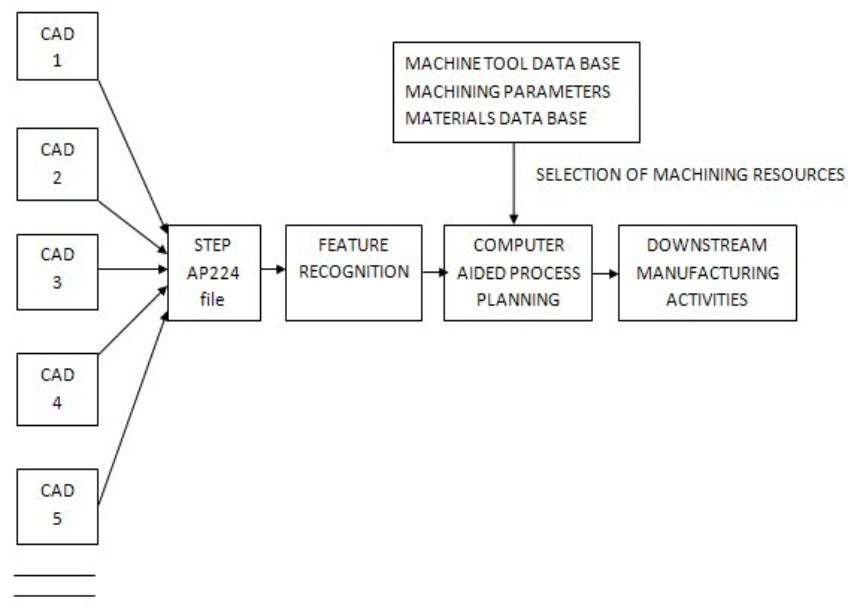

Fig. 1 Architecture of the system (Ashok G,et.al)

\section{DATABASE DESIGN}

The extracted data from the STEP file is stored in a database. This data further used for the development of process plan automatically. A database package has been used to design the database which is Microsoft access and is used only for testing purpose and simplicity. For commercial development, any rugged databases like oracle can be used. The database contains a database table called 'Feature Data' which has the following structure.

Table.1. Structure of database table

\begin{tabular}{|c|c|c|}
\hline \multicolumn{3}{|c|}{ Table name: Feature Data } \\
\hline $\begin{array}{l}\text { Variable } \\
\text { name }\end{array}$ & $\begin{array}{l}\text { Data } \\
\text { type }\end{array}$ & Description \\
\hline Entity & Text & Stores the name of the entity \\
\hline para1 & Text & Stores the first parameter of entity \\
\hline para2 & Text & $\begin{array}{l}\text { Stores the second parameter of } \\
\text { entity }\end{array}$ \\
\hline para3 & Text & Stores the third parameter of entity \\
\hline para4 & Text & $\begin{array}{l}\text { Stores the fourth parameter of } \\
\text { entity }\end{array}$ \\
\hline
\end{tabular}

In the above database table, the data type for all variables is assigned 'text' and when ever required the numeric parameters will be converted to numbers and vice versa.

\section{THROUGH SLOT FEATURE DETAILS}

\section{EXTRACTION}

The CAD model of a part is shown in Fig.1, consisting of a machining feature through slot, which is to be recognized from neutral file STEP AP-224 of the part for the details i,e slot width, length, height and angles of the sides of the slot with orientation of slot and its location. A computer program has been written to recognize the feature and its details in Visual Basic 6.0 based on the algorithm (procedure) developed. The following section will give details of the algorithm.
For identification of the feature, the necessary key data (parameters) related to feature should be extracted. The key data are, Cartesian point, direction and reference vectors, square_u_profile, swept shape, path feature component, linear path, SLOT(), Depth, Width, first_angle, second_angle, distance, first_radius, second_radius, etc. most of these parameters are shown in figure below.

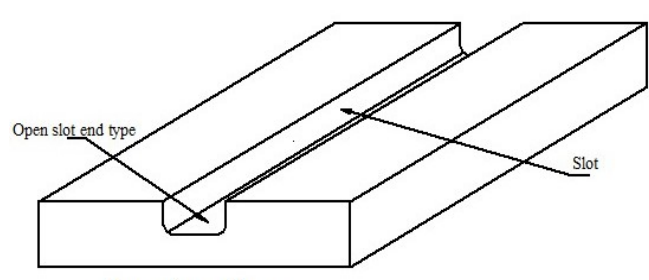

Open slot end type

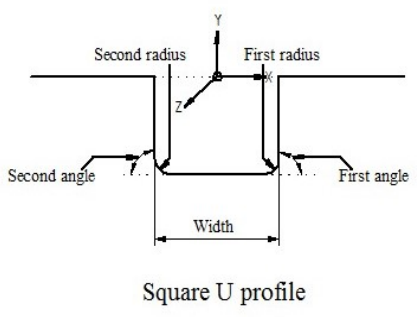

Fig.2 Open Slot end Type (Through Slot)

\section{ALGORITHM FOR RECOGNITION OF THROUGH SLOT FEATURE}

As mentioned above, for the identification of through slot feature, the necessary key parameters should be identified and extracted from the STEP A-P224 neutral file. The process of identification and extraction of the parameters along with their values is explained with sections of neutral file containing the respective parameters below.

Step 1: Read STEP AP-224 file from its ASCII form. The presence of any machining feature in the STEP AP-224 file is identified by occurrence of three entity, INSTANCED_FEATURE(), MACHINING_FEATURE() and actual feature occurrence like $\operatorname{SLOT}\left({ }^{-}\right), \operatorname{HOLE}()$, POCKET( ) etc., and these entities names are used as key words in identifying features. For example, the occurrence of any entity in the STEP file is as follows,

\section{\#...(CHARACTERIZED OBJECT()FEATURE DEFINITI ON()INSTANCED_FEATTUE()MACHINING_FEATURE ()FEATURE_NAME ()SHAPE_ASPECT());}

In the above STEP line, we can notice the presence of all the three entities, in place of actual feature name, it is mentioned as FEATURE_NAME (). In this place any of the feature names, which are defined in the STEP AP-224 application protocol, may appear. The features can also be identified by using only feature name as key word. In this research work, to identify the machining feature its name is used as key word in the format as defined in the STEP AP224.

Step 2: Search the STEP file for the presence of feature slot by using key word SLOT (), if the slot feature exist, then 
store the slot entity name I,e SLOT() in the data base. The STEP line which contains the feature name SLOT () appears as follows,

\section{\#...(CHARACTERIZED OBJECT()FEATURE DEFINITI ON()INSTANCED_FEATURE()MACHINING_FEATURE ()SLOT()SHAPE_ASPECT());}

Step 3: once the presence of feature SLOT () is confirmed, find next the parameters which identify the location of feature and its orientation. These can be identified by searching the entity AXIS2_PLACEMENT_3D () from STEP file making its name itself as key word as AXIS2_PLACEMENT_3D. the result of the search is shown below,

\#...=AXIS2_PLACEMENT 3D

('orientation',\#2001,\#2002,\#2003);

The entity AXIS2_PLACEMENT_3D ( ), contains four parameters, first parameter, the word 'orientation', second parameter is STEP line id or number pointing to Cartesian coordinate of slot feature representing its location, third parameter is again STEP line id pointing to direction vector and last parameter is again STEP line id pointing to another vector representing reference direction or orientation of the feature slot. Extract all these parameters and store in the database.

Step 4: Search for the id's or STEP lines which are identified as parameters of the entity AXIS2 _ PLACE MENT_3D ( ) in previous step I,e step 3, the result of the search is shown below,

\#2001=CARTESIAN_POINT ( " $(300.0,-30.0,15.0))$;

\#2002=DIRECTION (", $(-1.0,0.0,0.0))$;

\#2003=DIRECTION (",(0.0,-1.0,0.0));

Extract the parameters of the entity CARTESIAN_POINT () from STEP id \#2001, which has three parameters representing $\mathrm{x}, \mathrm{y}$ and $\mathrm{z}$ co-ordinates representing location of the feature and store it in database. Similarly extract the parameters of the two entities DIRECTION () found on STEP id \#2002 and \#2003. The first one represents the direction and second, reference direction which is the orientation of the feature slot. Store these $\mathrm{x}, \mathrm{y}$ and $\mathrm{z}$ parameter values of the entities in the database.

Step 5: A slot feature is made by sweeping a $U$ shaped geometric profile along a linear path. The $U$ shaped geometry profile is represented by entity SQUARE U_PROFILE () and the linear path is represented by entity PATH_FEATURE_COMPONENT (). Search for the two entities, the presence of these entities confirms the presence slot feature. Following is the search result for the two entities. Store these two entities in the database.

\#.... =SQUARE_U_PROFILE (', 'swept shape', \#2010,. F.); \#....=PATH_FEATURE_COMPONENT ('linear path', 'linear', \#2011 1,.F.);
The entity SQUARE U PROFILE () has four parameters, we are concern with the second parameter, which is a string 'swept shape'. Extract from the entity and store in the database. Similarly the entity PATH_FEATURE COMPON ENT () has four parameters, extract the first and second parameters, which are strings, 'linear path' and 'linear' respectively and store in database.

Step 6: The previous step confirms the presence of slot feature. Now we will find the other details of the slot. Depth of the slot is identified by searching two entities, REPRESENTATION_ITEM () and LENGTH_MEASURE () with parameters 'depth' and its magnitude from respective entities. Extract these data from the entities and store in the database. The search result and the corresponding entities are shown below.

\section{\#... $\quad($ LENGTH MEASURE WITH UNIT MEASURE REPRESENTATION ITEM - MEASURE_WITH_UNIT (LENGTH_MEASURE (10.0), \#15) QUALIFIED - REPRESENTATION_ITEM ((\#2015,\#2016,\#2017)) \\ REPRESENTATION_ITEM ('depth'));}

Step 7: The next parameter of the slot is to be identified is width of slot, width of the slot is identified by searching two entities, REPRESENTATION_ITEM () and LENGTH MEASURE () with parameters 'width' and its magnitude from respective entities. Extract these data from the entities and store in the database. The search result and the corresponding entities are shown below.

\section{\#...(LENGTH_MEASURE_WITH_UNIT( ) \\ MEASURE_REPRESENTĀTION_ITEM () MEASURE \\ _WITH_UNIT(LENGTH_MEASURE(25.0),\#15) \\ QUALIFIED_REPRESENTTATION_ITEM ((\#2158, \#2159, \#2160)) REPRESENTATION_ITEM ('width'));}

Step 8: The next parameter of the slot is to identify is 'first angle'. First angle is the angle between bottom surface and right vertical surface. This parameter is identified by searching two entities, REPRESENTATION_ITEM () and PLANE_ANG LE_MEASURE () with parameters 'first angle' and its magnitude from respective entities. Extract these data from the entities and store in the database. The search result and the corresponding entities are shown below.

\section{\#..=(MEASURE_REPRESENTATION_ITEM ()}

MEASURE_WITH_UNIT (PLANE_ANGLE_MEASURE (20.0),\#24) PLANE_ANGLE_MEASURE_WITH_UNIT () QUALIFIED_REPRESENTĀTION_ITEM $((\# 21 \overline{67}, \# 2168 \#$ 2169)) REPRESENTATION_ITEM ('first angle'));

Step 9: The next parameter of the slot to identify is 'second angle'. Second angle is the angle between bottom surface and left vertical surface of the slot. This parameter is identified by searching two entities, REPRESENTATION_ITEM () and PLANE_ANGLE_MEA SURE() with parameters 'second angle' and its magnitude from respective entities. Extract these data from the entities 
and store in the database. The search result and the corresponding entities are shown below.

\#...=(MEASURE_REPRESENTATION_ITEM ()

MEASURE_WITH_UNIT (PLANE_A ANGLE_MEASURE (90.0),\#24)

PLANE_ANGLE_MEASURE_WITH_UNIT()

QUALIFIED_REPRESENTATION_ITEM((\#2176,\#2177,\#

2178)) REPRESENTATION_ITEM ('second angle'));

Step 10: The next parameter of the slot is to identify is first radius. First radius is the radius of arc between bottom surface and right vertical surface. This parameter is identified by searching two entities, REPRESENTATION_ITEM () and LENGTH_MEASURE () with parameters 'first radius' and its magnitude from respective entities. Extract these data from the entities and store in the database. The search result and the corresponding entities are shown below.

\#... $=($ LENGTH_MEASURE_WITH_UNIT()MEASURE_R EPRESENTATION_ITEM)

MEASURE_WITH_UNIT(LENGTH_MEASURE (0.0) , \#15) REPRĒESNTĀTION_ITEM ('first radius')) ;

Step 11: The next parameter of the slot is to identify is 'second radius. Second radius is the radius of arc between bottom surface and left vertical surface. This parameter is identified by searching two entities, REPRESENTATION_ITEM () and LENGTH_MEASURE () with parameters 'second radius' and its magnitude from respective entities. Extract these data from the entities and store in the database. The search result and the corresponding entities are shown below.

$\# . .=\quad$ (LENGTH_MEASURE_WITH_UNIT $\quad(\quad)$ MEASURE_REPRESENTATION $\bar{N}$ ITEM () MEASURE_WITH_UNIT (LENGTH_MEASURE ( 0.0$)$, \#15) REPRESENTĀTION_ITEM ('second radius'));

Step 12: The next parameter of the slot is to identify is 'distance'. The 'distance' is the length of the slot. This parameter is identified by searching two entities, REPRESENTATION_ITEM () and LENGTH_MEASURE () with parameters 'distance' and its magnitude from respective entities. Extract these data from the entities and store in the database. The search result and the corresponding entities are shown below.

\#... $=$

(LENGTH_MEASURE_WITH_UNIT()

MEASURE REPRESENTATION ITEM () MEASURE

WITH_UNIT (LENGTH_MEATSURE (300.0), \#15) QUALIFIED_REPRESE $\bar{N}$ TATION_ITEM ((\#2203, \#2204, \#2205)) REPRESENTATIONS_ITE-M ('distance'));

Sep 13: The last parameter of the slot to be identify is related to end condition of the slot, i,e whether the slot is 'blind (closed)' or 'open (through)'. This parameter is identified by searching entity, SLOT_END (). This entity has four parameters; the second parameter of the entity provides the information related to end condition of the slot.
Either 'open' or 'closed', extract this parameter and store in the database. The search result and the corresponding entities are shown below.

\#....=SLOT END ('", 'open-, \#2220, F.)

The output of the above procedure or algorithm is shown below in the database table,

Table.2. Database table showing extracted data from STEP file

\begin{tabular}{|l|l|l|l|l|}
\hline Entity & para1 & para2 & para3 & para4 \\
\hline Slot & Null & Null & Null & \\
\hline $\begin{array}{l}\text { Axis__placement } \\
\text { 3d }\end{array}$ & 'orientation' & $\# 2001$ & $\# 2002$ & $\# 2003$ \\
\hline Cartesian_point & 300.0 & -30.0 & 15.0 & \\
\hline Direction & -1.0 & 0.0 & 0.0 & \\
\hline Direction & 0.0 & -1.0 & 0.0 & \\
\hline Square_u_profile & $\begin{array}{l}\text { 'swept } \\
\text { shape' }\end{array}$ & & & \\
\hline $\begin{array}{l}\text { Path_feature_ } \\
\text { Component }\end{array}$ & 'linear path' & 'linear' & & \\
\hline Representation_item & 'depth' & 10.0 & & \\
\hline Representation_item & 'width' & 20.0 & & \\
\hline Representation_item & 'first angle' & 90.0 & & \\
\hline Representation_item & $\begin{array}{l}\text { 'second } \\
\text { angle' }\end{array}$ & 90.0 & & \\
\hline Representation_item & 'first radius' & 0.0 & & \\
\hline Representation_item & $\begin{array}{l}\text { 'second } \\
\text { radius' }\end{array}$ & 0.0 & & \\
\hline Representation_item & 'distance' & 300.0 & & \\
\hline Slot_end & 'open' & & & \\
\hline
\end{tabular}

\section{CONCLUSION}

The feature recognition is one of the key activity in developing a Computer Aided Process Planning (CAPP). This research work is a part of development CAPP system. The algorithm or procedure developed for recognition of slot feature from a neutral file STEP AP-224 of CAD model can be implemented by using any of the computer language which can easily interact with database. The program written is based on the algorithm developed, will recognize the feature from STEP AP-224 neutral file of the CAD model and extract the entities and data related to through slot feature and store it in a database designed for the purpose. This stored data is further used for the development of CAPP system.

\section{REFERENCES}

[1] S. Nagarajan \& N. Venkata Reddy , 2010, STEPbased automatic system for recognising design and manufacturing features, International Journal of Production Research, 48:1, 117-144.

[2] Patrick Martin \& Alain D'Acunto, 2007, Method of determining the process applied for feature machining: experimental validation of a slot, International Journal of Advanced Manufacturing Technology, Vol. 37, no 9-10, p.898-907. 
[3] Jung Hyun Han and Inho Han and Eunseok Lee and Juneho Yi, 2001, Manufacturing feature recognition toward integration with process planning, IEEE Transactions on Systems, Man, and CyberneticsPart B: Cybernetics, Vol.31, 373.

[4] Jaider Oussama, Elmesbahi Abdelilah, Rechia Ahmed, May 2014, Manufacturing Computer Aided Process Planning For Rotational Parts. Part 1: Automatic Feature Recognition from STEP AP203, Int. Journal of Engineering Research and Application ISSN : 2248-9622, Vol. 4, Issue 5( Version 6), pp.1425.

[5] Hyun Chan Lee and Wan Chul Jhee and Hee Sok Park, 2007, Generative CAPP through projective feature recognition, Computers \& Industrial Engineering, Vol.53, 241-246.

[6] Tan C F and Ismail N and Wong S V and Sulaiman S and Osman M R, 2005, Development of Hole Recognition System from Step File, AJSTD, Vol.22, 285-295.

[7] Chen Wong Keong, a, Yusri Yusof, 2012, Developing a Feature-based System for Automated Machining Feature Recognition (ISO 10303 AP 224) of Prismatic Components, Applied mechanics and materials, volume 229

[8] Sreenivasulu Reddy.A1, PoornachandraSekhar.Y2, Rajendra Prasad.T3 , Ravindranath.K, December12th-14th, 2014, Design and development of feature extraction and recognition information system for computer aided process planning systems, 5 th International \& 26th All India Manufacturing Technology, Design and Research Conference (AIMTDR 2014) IIT Guwahati, Assam, India.

[9] Arunkumar P and Anand Deshpande S and Kumar A C S, 2008, A System for Extracting Product Features from CAD Models - A STEP Approach, Contemporary Engineering Sciences, Vol.1, 139 146.

[10] Jami J. Shah, David Anderson, Yong Se Kim, Sanjay Joshi, MARCH 2001, A Discourse on Geometric Feature Recognition From CAD Models, Journal of Computing and Information Science in Engineering, Vol. $1 / 41$.

[11] V. N. Malleswaria*, Dr. P. M.Vallib , Dr. M. M. M. Sarcarc, March-2013, Automatic Recognition of Machining Features using STEP Files, International Journal of Engineering Research \& Technology (IJERT) Vol. 2 Issue 3.

[12] D. Sreeramulu1, C.S.P.Rao2, 2011, A new methodology for recognizing features in rotational parts using STEP data exchange standard, International Journal of Engineering, Science and Technology, Vol. 3, No. 6, pp. 102-115.

[13] Yingguang Li, Wei Wang, Xu Liu \& Yongsheng Ma, 2014, Definition and recognition of rib features in aircraft structural part, International Journal of Computer Integrated Manufacturing, 27:1, 1-19

[14] Grzegorz Nikiel, 2010, geometrical data extraction of axisymmetric parts from the b-rep models, Advances in manufacturing science and technology, Vol. 35, No. 2,
[15] Ashok G, Dr.S S Hebbal, Dr.Scachhidanand Reur, 2015, Recognition of Hole Feature and Stock Details of a Prismatic Part from Its STEP AP224 Neutral File. 